9. - On nonlinear P-compact operators in Banach space with applications to constructive fixed point theorems, J. Math. Anal Appl. (to appear).

10. E. Rothe, Zur Theorie der topologischen Ortnung und der Vektorfelder in Banachschen Raumen, Compositio Math. 5 (1937), 177-197.

11. J. Schauder, Der Fixpunksatz in Functionalraumen, Studia Math. 2 (1930), 171-180.

12. M. Shinbrot, $A$ fixed point theorem and some applications, Arch. Rational. Mech. Anal. 17 (1964), 255-271.

University of Chicago

\title{
A PERTURBATION LEMMA
}

\section{BY KARL GUSTAFSON ${ }^{1}$}

Communicated by F. Browder, September 29, 1965

1. Introduction. We will prove the following lemma and investigate some of its implications: namely, a short proof by Goldberg [1] of the basic perturbation theorem of Kato [2], avoiding previous homotopy arguments; an extension of results of Trotter and Nelson [3] for semigroup generators; and a criterion for well-posed perturbed problems in spaces that are not necessarily complete. For further references and more information, see [1], [2], and [3].

Throughout this paper all operators are linear with domains subspaces of a normed linear space $X$ and ranges subspaces of a normed linear space $Y$. If an operator $B$ perturbs an operator $T$, we assume that $D(B) \supset D(T)$.

In this section, the spaces need not be complete.

Lemma 1. Let $T^{-1}$ and $B$ be bounded operators with $\|B\|<\left\|T^{-1}\right\|^{-1}$. Then

$$
\operatorname{dim} Y / \mathrm{Cl}(R(T))=\operatorname{dim} Y / \mathrm{Cl}(R(T+B)) .
$$

Proof. ${ }^{2}$ We use the known result (e.g., see [1] for a proof) that if $\|B\|<\left\|T^{-1}\right\|-1$, then

$$
\operatorname{dim} Y / \mathrm{Cl}(R(T+B)) \leqq \operatorname{dim} Y / \mathrm{Cl}(R(T)) .
$$

1 Partially supported by a NATO postdoctoral fellowship.

2 Concerning this little result, let $\|B\|<\alpha\left\|T^{-1}\right\|^{-1}$. The author appreciates discussions with Dr. Seymour Goldberg, who proved it for $\alpha=1 / 2$ in his lectures. The main trick in the proof can be seen for the case $\alpha=3 / 4$. The author also appreciates the aid of $\mathrm{Mr} \mathrm{J}$. Kuttler in extending the result from $\alpha=3 / 4$ to $\alpha=7 / 8$. 
From (1.2) we see that the proof of (1.1) reduces to showing

$$
\operatorname{dim} Y / \mathrm{Cl}(R(T)) \leqq \operatorname{dim} Y / \mathrm{Cl}(R(T+B)) .
$$

The trick in showing (1.3) is to perturb and unperturb $T$ successively by fractions of $B$ of just the right size, using (1.2) at each stage.

We first note that $\|B\|<\left\|T^{-1}\right\|^{-1}$ implies that there exists some integer $n>0$ such that $\|B\|<\left.\left[\left(2^{n}-1\right) / 2^{n}\right] \cdot\left\|T^{-1}\right\|\right|^{-1}$. We now let $c_{k}=\left(2^{n-k}\right) /\left(2^{n}-1\right)$ for $k=1, \cdots, n$. For convenience, we also let $c_{0}=0$. We now claim that

$$
\begin{aligned}
\operatorname{dim} Y / \mathrm{Cl}\left(R\left[T+\left(\sum_{k=0}^{m-1} c_{k}\right) B\right]\right) & \\
& \leqq \operatorname{dim} Y / \mathrm{Cl}\left(R\left[T+\left(\sum_{k=0}^{m} c_{k}\right) B\right]\right)
\end{aligned}
$$

for each $m=1, \cdots, n$. We use (1.2), with a perturbation by $-c_{m} B$, to show that (1.4) holds, since

$$
\begin{aligned}
\left\|-c_{m} B\right\| & <\left[\left(2^{n-m}\right) /\left(2^{n}-1\right)\right] \cdot\left[\left(2^{n}-1\right) / 2^{n}\right] \cdot\left\|T^{-1}\right\|-1 \\
& =\left.2^{-m}\left\|T^{-1}\right\|\right|^{-1}
\end{aligned}
$$

and noting that $\sum_{k=0}^{m} c_{k}=\left[2^{n} /\left(2^{n}-1\right)\right] \cdot\left[\left(2^{m}-1\right) / 2^{m}\right]$, we have for $x \in D(T)$

$$
\begin{aligned}
\frac{\left\|\left(T+\left(\sum_{k=0}^{m} c_{k}\right) B\right) x\right\|}{\|x\|} & \geqq \frac{\|T x\|}{\|x\|}-\left(\sum_{k=0}^{m} c_{k}\right) \frac{\|B x\|}{\|x\|} \\
& >\left.\left[1-\left(2^{m}-1\right) / 2^{m}\right] \cdot\left\|T^{-1}\right\|\right|^{-1} \\
& =2^{-m}\left\|T^{-1}\right\|-1
\end{aligned}
$$

From (1.5) and (1.6) we have $\left\|-c_{m} B\right\|<\left.\left\|\left(T+\left(\sum_{k=0}^{m} c_{k}\right) B\right)^{-1}\right\|\right|^{-1}$, which by (1.2) is sufficient for (1.4) to hold for each $m=1, \cdots, n$. Combining these $n$ inequalities then yields (1.3).

2. Perturbation theory. In this section we assume that both $X$ and $Y$ are complete spaces. We first state some known definitions.

An operator $T$ is called normally solvable (n.s.) if it is closed and has closed range. If the kernel of $T$ is closed, the minimum modulus is defined by $\gamma(T)=\inf _{x \in D(T), x \notin N(T)}[\|T x\| / d(x, N(T))]$. We also have the three indices $\alpha(T)=\operatorname{dim} N(T), \beta(T)=\operatorname{dim} Y / R(T)$, and if either $\alpha<\infty$ or $\beta<\infty, \kappa(T)=\alpha(T)-\beta(T)$.

Goldberg [1] has employed Lemma 1 to give a short proof of a 
perturbation theorem of Kato [2]; roughly, that if $T$ is n.s. and $B$ is bounded and small, then $T+B$ is n.s. and certain relations hold between the indices of $T$ and those of $T+B$. We now state for future reference the known extended version of this theorem, wherein $B$ is only required to be $T$-bounded.

Theorem (Perturbation). Let $T$ be n.s. and possess index $\kappa$. Let $B$ satisfy

$$
\|B x\| \leqq a\|T x\|+b\|x\|
$$

for all $x \in D(T)$, where $b+a \gamma(T)<\gamma(T)$. Then $T+B$ is n.s., $\alpha(T+B)$ $\leqq \alpha(T), \beta(T+B) \leqq \beta(T)$, and $\kappa(T+B)=\kappa(T)$.

To illustrate the role that Lemma 1 can play in such a context, we note that if $T$ also possesses a bounded inverse and $\|B\|<\gamma(T)$ $\equiv\left\|T^{-1}\right\|{ }^{-1}$, Lemma 1 states that

$$
\kappa(T)=-\beta(T)=-\beta(T+B)=\kappa(T+B) .
$$

3. Semigroup generators. We now prove the following:

THEOREM 2. Let $A$ be the infinitesimal generator of a contraction semigroup on the Banach space $X$, and let $B$ be a dissipative operator with $D(B) \supset D(A)$. If there exist constants $a$ and $b$, with $a<1$, such that for all $\phi \in D(A)$,

$$
\|B \phi\| \leqq a\|A \phi\|+b\|\phi\|,
$$

then $A+B$ is the infinitesimal generator of a contraction semigroup.

REMARK. The above result, as an extension of an earlier result by Trotter, is obtained by Nelson [3] under the additional condition that $a<\frac{1}{2}$. For definitions, references, and applications to the Schrödinger equation and semigroup generation, see [3]. For our purposes, we will use: (i) Nelson's form of the Hille-Yosida-Phillips Theorem, characterizing a densely defined operator $T$ as the infinitesimal generator of a contraction semigroup if and only if it is dissipative and there exists $\lambda_{0}$ such that $\lambda>\lambda_{0}$ implies that $\lambda$ is in the resolvent set of $T$; (ii) any dissipative operator $T$ satisfies $\|(\lambda-T) \phi\| \geqq \lambda\|\phi\|$ for all $\phi \in D(T)$ and all real $\lambda$; and (iii) the dissipative operators form a convex cone.

Proof of Theorem 2. By the above remark, $A+B$ is dissipative and $(\lambda-A-B)^{-1}$ is continuous for any positive $\lambda$. The remainder of the proof thus consists of showing the existence of some $\lambda_{0}$ such that for $\lambda>\lambda_{0}, R(\lambda-A-B)=X$. 
We will first show that for $a<\frac{1}{2}$, Theorem 2 is a direct corollary of the above-stated perturbation theorem. Then by a device motivated by Lemma 1 , we will extend the result to $a<1$.

Suppose $a<\frac{1}{2}$. Then for any operator $A$ and positive $\lambda$,

$$
a\|A \phi\|+b\|\phi\| \leqq a\|(\lambda-A) \phi\|+(a \lambda+b)\|\phi\| .
$$

Now let $\lambda>\lambda_{0}=\max \left\{\lambda_{0}(A), b /(1-2 a)\right\}$. Then since $A$ is dissipative,

$$
\begin{aligned}
b^{\prime}+a \gamma(\lambda-A) & \equiv(a \lambda+b)+\left.a\left\|(\lambda-A)^{-1}\right\|\right|^{-1} \\
& <(1-a) \lambda+\left.a\left\|(\lambda-A)^{-1}\right\|\right|^{-1} \\
& \leqq\left.(1-a)\left\|(\lambda-A)^{-1}\right\|\right|^{-1}+a\left\|(\lambda-A)^{-1}\right\|-1 \\
& =\gamma(\lambda-A),
\end{aligned}
$$

which by the perturbation theorem and $A$ 's properties yields that

$$
R(\lambda-A-B)=R(\lambda-A)=X .
$$

Suppose $\frac{1}{2} \leqq a<1$. Then $a<\left(2^{m}-1\right) / 2^{m}$ for some integer $m$. Let $\alpha=2^{m-1} /\left(2^{m}-1\right)$ and note that $\alpha a<\frac{1}{2}$. From (3.1) we have

$$
\alpha\|B \phi\| \leqq \alpha a\|A \phi\|+\alpha b\|\phi\|
$$

and thus $A+\alpha B$ is the infinitesimal generator of a contraction semigroup. From (3.1) we also have

$$
\begin{aligned}
& \alpha\|B \phi\| \leqq \alpha a\left\|\left(A+\alpha\left(\sum_{j=0}^{k-1} 2^{-j}\right) B\right) \phi\right\| \\
& +\frac{1}{2} \alpha\left(\sum_{j=0}^{k-1} 2^{-j}\right)\|B \phi\|+\alpha b\|\phi\|
\end{aligned}
$$

for $k=1, \cdots, m-1$. Since $\sum_{j=0}^{k-1} 2^{-j}=\left(2^{k}-1\right) /\left(2^{k-1}\right)$, (3.6) gives

$$
2^{-k} \alpha\|B \phi\| \leqq \alpha a\left\|\left(A+\alpha\left(\sum_{j=0}^{k-1} 2^{-j}\right) B\right) \phi\right\|+\alpha b\|\phi\|,
$$

which at each step yields $\left[A+\alpha\left(\sum_{j=0}^{k} 2^{-i}\right) B\right]$ as an infinitesimal generator of a contraction semigroup, which for $k=m-1$ is the desired result for $A+B$.

CoROllary 3. Under the conditions of Theorem 2, except with $a<a_{1}$, $c(A+d B)$ is the infinitesimal generator of a contraction semigroup for all $c \geqq 0$ and all $0 \leqq d \leqq 1 / a_{1}$.

4. Well-posed problems. Since Lemma 1 holds for spaces that are not necessarily complete, it would appear to be useful in other ways, 
such as the following inference of well-posed (uniqueness, stability, existence) perturbations from well-posed base problems.

EXAMPLE 4. Let $T^{-1}$ and $B$ be bounded, $\|B\|<\left\|T^{-1}\right\|-1$, and $R(T)$ $=Y$. If $R(T+B)$ is closed, then the equation

$$
(T+B) x=y
$$

is well-posed.

Proof. The uniqueness and stability follow from (1.6) when $m=n$ there. The existence of a solution follows from (1.1) and $R(T+B)$ closed, which imply that $R(T+B)=Y$.

Although the main feature of the example is that the spaces need not be complete, we may observe that if $X$ is complete, the conclusion of the example is

$$
R(T+B) \text { closed } \Leftrightarrow T+B \text { closed } \Leftrightarrow \text { well-posed (4.1) }
$$

Furthermore, the hypotheses of the example imply that $T$ is closed; hence if $D(B)$ is closed, it follows that $T+B$ is closed.

\section{REFERENCES}

1. Seymour Goldberg, Unbounded linear operators with applications, McGrawHill, New York (to appear).

2. T. Kato, Perturbation theory for nullity, deficiency, and other quantities of linear operators, J. Analyse Math. 6 (1958), 261-321.

3. Edward Nelson, Feynman integrals and the Schrödinger equation, J. Mathematical Phys. 5 (1964), 332-343.

UNIVERSITY OF MARYLAND AND

Battelle Institute, Geneva, Switzerland 